\title{
Effect of variety and water management on the growth and yield of Boro rice
}

\author{
S Murshida ${ }^{1}$, MR Uddin ${ }^{1 *}$, MP Anwar ${ }^{1}$, UK Sarker ${ }^{1}$, MM Islam $^{2}$, MMI Haque ${ }^{3}$
}

${ }^{1}$ Department of Agronomy, Bangladesh Agricultural University, Mymensingh 2202, Bangladesh; ${ }^{2}$ Agriculture

Training Institute, Rangamati, Bangladesh: ${ }^{3} \mathrm{ACI}$ Limited, Dhaka, Bangladesh

\begin{abstract}
An experiment was conducted at the Agronomy Field Laboratory, Bangladesh Agricultural University, Mymensingh during December 2014 to May 2015 to examine the effect of variety and water management system on the growth and yield performance of boro rice. The experiment consisted of three varieties (cv. BRRI dhan28, BRRI dhan29 and Binadhan-14) and four water management systems (viz. Traditional flooding, non-flooded rice straw mulching, non-flooded water hyacinth mulching and non-flooded no mulching). The experiment was laid out in a split plot design with three replications. Different growth characters, yield and yield contributing characters of boro rice were found to the significantly influenced by variety, water management system and their interactions. At 100 DAT, the highest plant height, maximum number of tillers hill ${ }^{-1}$, dry matter of shoot hill ${ }^{-1}$ and dry matter of root hill ${ }^{-1}$ were obtained from BRRI dhan29 and the lowest values were found in Binadhan-14. At 100 DAT, the highest plant height, maximum number of tillers hill ${ }^{-1}$, dry matter of shoot hill ${ }^{-1}$ and dry matter of root hill ${ }^{-1}$ were obtained in nonflooded rice straw mulching treatment and the lowest ones were obtained from non-flooded no mulching treatment. Variety had significant effect on all the crop characters under study except 1000-grain weight. The highest grain yield was obtained from BRRI dhan29 and the lowest value was recorded from Binadhan-14. Water management system was also significantly influenced all crop characters. The highest grain yield was recorded from non-flooded rice straw mulching treatment and the lowest grain yield was found from non-flooded no mulching treatment. The interaction of variety and water management system showed that BRRI dhan29 with non-flooded rice straw mulching resulted in the highest grain yield whereas the lowest yield was observed from the interaction of Binadhan-14 with non-flooded no mulching treatment. The result of the experiment suggests that BRRI dhan29 can be grown economically with non-flooded rice straw mulching treatment.
\end{abstract}

Key words: Rice, water management, traditional flooding, mulching

Progressive Agriculturists. All rights reserve

*Corresponding Author: romijagron@yahoo.com

\section{Introduction}

The population of Bangladesh is still growing by two million every year and may increase by another 30 million over the next 20 years. Thus, Bangladesh will require about 57.26 million tons of rice for the year 2020 (BRRI, 2011). To reach the goal, it is necessary either to increase the crop area or to increase yield per unit area. But due to high population pressure, horizontal expansion of land is not possible. Therefore, increasing yield per unit area is the only means. In Bangladesh, there are three diverse growing seasons of rice namely, aus, aman and boro. Among different rice groups of Bangladesh boro is the most important that covers an area of 4.72 million hectares with a production of 13.73 million tons of grains (BBS, 
2011).Variety is one of the most important factors to be considered for getting increased rice production. Use of high yielding varieties and hybrid varieties in Bangladesh has been increased remarkably in recent years and the country has almost reached a level of self sufficiency in rice production. Selection of potential variety, planting in appropriate method and application of optimum amount of plant nutrients and optimum insistence can play important role in increasing yield and national income. In Bangladesh about $4.065 \mathrm{~m}$ ha land is under boro rice cultivation of which modern high yielding varieties (MV) cover about $96 \%$, most of them are with irrigation. Irrigation is one of the costly inputs of boro rice cultivation in Bangladesh. The MVs that are recommended for boro season have high yield potential and they require huge amount of irrigation water and without much irrigation they cannot produce desired yield. It is an established fact that for producing $1 \mathrm{~kg}$ of paddy rice, it requires even up to 2500 liters of water (De Silva, 2007). Thus irrigation is imparting alarming rise of cost of production along with other inputs. Recently management practice like AWD (Alternate wetting and drying) is suggested to reduce irrigation water requirement. Insufficient water affects the uptake of nutrients from soil. Nutrients from soil reach the root surface by mass flow and diffusion process which are related to moisture content of the soil. Movement of nutrients through the plant body by physiological activities is also associated with soil water (Tisdale et al., 1985). But as a matter of fact, irrigation water in Bangladesh is limited resource and hence irrigation practices must be rationalized for high water use efficiency. Application of irrigation water without proper planning based on actual requirement of the crops results not only its wastages but also hampers crop growth and yield (Sekhon et al., 1986). Knowledge on the role of irrigation in growth and development of plant and the optimum time of application of water may help to economize the use of limited amount of water in obtaining the maximum yield of rice. Irrigation frequency has a significant influence on the growth and yield of rice. With the increasing of irrigation frequencies the grain yield of rice can be increased. Therefore the present study was undertaken to evaluate the performance of three boro rice varieties, to find out suitable water management system for boro rice cultivation and to verify the effect of interaction (if any) in between variety and water management system on the performance of boro rice.

\section{Materials and Methods}

A field experiment was carried out at the Agronomy Field laboratory, Bangladesh Agricultural University (BAU), Mymensingh during the period from December 2014 to May 2015 in order to study the growth and yield of boro rice as affected by water management system. The experimental consisted of the following treatments. i) Rice Cultivars viz. BRRI dhan28 $\left(\mathrm{V}_{1}\right)$, BRRI dhan29 $\left(\mathrm{V}_{2}\right)$ and Binadhan-14 $\left(\mathrm{V}_{3}\right)$ ii) Water management systems viz. Traditional flooding $\left(\mathrm{M}_{1}\right)$, Non-flooded rice straw mulching $\left(\mathrm{M}_{2}\right)$, Non-flooded water hyacinth mulching $\left(\mathrm{M}_{3}\right)$ and Non-flooded no mulching $\left(\mathrm{M}_{4}\right)$. The experiment field was Noncalcareous Dark Grey Floodplain under the AgroEcological Region of the Old Brahmaputra FloodplainAEZ9 (UNDP and FAO, 1988).The experiment was laid out in a split-plot design with three replications. Water management treatments were assigned to the main plot and variety to the sub plot, respectively, at random. The size of a unit plot was $10 \mathrm{~m}^{2}(4 \mathrm{~m} \times 2.5 \mathrm{~m})$. The number of plot is 36 . During final land preparation each unit plot was fertilized with Triple Super Phosphate (TSP), Muriate of Potash (MOP), Gypsum and $\mathrm{ZnSO}_{4} @$ 90,60, 20 and $5 \mathrm{~kg} \mathrm{ha}^{-1}$ respectively. Urea was applied@300 kg ha ${ }^{-1}$ in three installments at 15, 30 and 45 days after transplanting (DAT). All management practices were done as and when necessary. Harvesting was done when $90 \%$ of the grains became golden (Binadhan-14), golden yellow (BRRI dhan29), fading golden (BRRI dhan28) in color. Five hills (excluding border hills) were selected randomly from each harvested plot to record the crop characters and yield components. After recording data, the grains and straws of each the sampling were added 
to the total grain and straw yields of respective plots. The harvested crops of each plot were separately bundled, tagged and then brought to threshing floor. Threshing was done by pedal thresher. After threshing the grains were cleaned and sun dried to maintain moisture content of $14 \%$. Straws were also dried properly. Finally the grain and straw yields were recorded and converted to $\mathrm{t} \mathrm{ha}^{-1}$.Data collected on different parameters were statistically analyzed using a software, named MSTAT. Mean comparisons of the effect of treatment and interactions from the analysis of variance (ANOVA) were made by Duncan's Multiple Range Test (Gomez and Gomez, 1984).

\section{Results and Discussion}

\section{Growth performance of boro rice}

Effect of variety: Plant height, number of tillers hill ${ }^{-1}$, dry matter of shoot hill ${ }^{-1}$ and dry matter of root hill ${ }^{-1}$ were significantly influenced by variety. The highest plant height $(90.27 \mathrm{~cm})$, number of total tillers hill ${ }^{-1}$ (7.24), dry matter of shoot hill ${ }^{-1}(26.38 \mathrm{~g})$ and dry matter of root hill' ${ }^{-1}(11.00 \mathrm{~g})$ were found in BRRI dhan29 at 100 DAT and the lowest plant height, number of tillers hill ${ }^{-1}$, dry matter of shoot hill ${ }^{-1}$ and dry matter of root hill ${ }^{-1}$ was found in Binadhan-14 at all sampling dates (Table $1 \& 4$ ). Variable effect of variety on plant height was also reported by Om et al. (1998) and Krisna (2002) who also recorded variable plant height among varieties. Variable effect of variety on number of total tillers hill ${ }^{-1}$ was also reported by BINA (1998) and Nuruzzaman et al. (2000) who noticed that number of total tillers hill ${ }^{-1}$ differed among varieties. These might be due to genetic make-up of the varieties.

Effect of water management system: Water management system significantly influenced plant height, number of tillers hill ${ }^{-1}$, dry matter of shoot hill ${ }^{-1}$ and dry matter of root hill ${ }^{-1}$ at all sampling dates. The highest plant height $(82.15 \mathrm{~cm})$, number of tillers hill ${ }^{-1}$ (7.07), dry matter of shoot hill $^{-1}(27.71 \mathrm{~g})$ and dry matter of root hill $^{-1}(11.47 \mathrm{~g})$ were produced by nonflooded rice straw mulching $\left(\mathrm{M}_{2}\right)$ treatment and the lowest plant height, number of total tillers hill ${ }^{-1}$, dry matter of shoot hill ${ }^{-1}$ and dry matter of root hill ${ }^{-1}$ was produced by non-flooded no mulching $\left(\mathrm{M}_{4}\right)$ treatment (Table $2 \& 5$ ).

Interaction effect of variety and water management system: The data showed that the plant height and number of tillers hill ${ }^{-1}$ was non-significant for all sampling dates except for 100 DAT (Table 3).

Table 1. Effect of variety on growth of boro rice at different dates after transplanting (DAT)

\begin{tabular}{ccccc|cccc}
\hline \multirow{3}{*}{ Variety } & \multicolumn{4}{c|}{ Plant height (cm) } & \multicolumn{4}{c}{ No of tillers hill $^{-1}$} \\
\cline { 2 - 8 } & $\mathbf{2 5}$ & $\mathbf{5 0}$ & $\mathbf{7 5}$ & $\mathbf{1 0 0}$ & $\mathbf{2 5}$ & $\mathbf{5 0}$ & $\mathbf{7 5}$ & $\mathbf{1 0 0}$ \\
& DAT & DAT & DAT & DAT & DAT & DAT & DAT & DAT \\
\hline $\mathrm{V}_{1}$ & $28.76 \mathrm{~b}$ & $54.42 \mathrm{~b}$ & $72.60 \mathrm{~b}$ & $80.07 \mathrm{~b}$ & $6.56 \mathrm{ab}$ & $6.44 \mathrm{ab}$ & $6.56 \mathrm{ab}$ & $6.40 \mathrm{ab}$ \\
$\mathrm{V}_{2}$ & $38.12 \mathrm{a}$ & $62.61 \mathrm{a}$ & $79.26 \mathrm{a}$ & $90.27 \mathrm{a}$ & $6.95 \mathrm{a}$ & $6.84 \mathrm{a}$ & $6.95 \mathrm{a}$ & $7.24 \mathrm{a}$ \\
$\mathrm{V}_{3}$ & $23.52 \mathrm{c}$ & $50.10 \mathrm{c}$ & $69.40 \mathrm{c}$ & $68.52 \mathrm{c}$ & $5.84 \mathrm{~b}$ & $5.80 \mathrm{~b}$ & $5.84 \mathrm{~b}$ & $6.06 \mathrm{~b}$ \\
$\mathrm{CV}(\%)$ & 6.05 & 8.94 & 2.17 & 2.75 & 7.14 & 12.74 & 7.25 & 11.42 \\
Level of & & & & & & & $*$ & $*$ \\
significance & $* *$ & $* *$ & $* *$ & $* *$ & $* *$ & $*$ & $* *$ & $* *$ \\
\hline
\end{tabular}

Here, in a column figures with same letters or without letter do not differ significantly whereas figures with dissimilar letter differ significantly as per DMRT. $\mathrm{V}_{1}=\mathrm{BRRI}$ dhan28, $\mathrm{V}_{2}=\mathrm{BRRI}$ dhan29, $\mathrm{V}_{3}=$ Binadhan-14, ${ }^{* *}=$ Significant at $1 \%$ level of probability, ${ }^{*}=$ Significant at $5 \%$ level of probability, DAT $=$ Days after transplanting 
Table 2. Effect of water management system on growth of boro rice at different dates after transplanting (DAT)

\begin{tabular}{ccccc|cccc}
\hline \multirow{2}{*}{$\begin{array}{c}\text { Water } \\
\text { management }\end{array}$} & \multicolumn{4}{c|}{ Plant height (cm) } & \multicolumn{4}{c}{ No of tillers hill $^{-1}$} \\
\cline { 2 - 9 } system & $\mathbf{2 5}$ & $\mathbf{5 0}$ & $\mathbf{7 5}$ & $\mathbf{1 0 0}$ & $\mathbf{2 5}$ & $\mathbf{5 0}$ & $\mathbf{7 5}$ & $\mathbf{1 0 0}$ \\
& DAT & DAT & DAT & DAT & DAT & DAT & DAT & DAT \\
\hline $\mathrm{M}_{1}$ & $29.75 \mathrm{c}$ & $54.04 \mathrm{c}$ & $72.58 \mathrm{c}$ & $78.63 \mathrm{c}$ & $6.35 \mathrm{~b}$ & 6.36 & $6.35 \mathrm{c}$ & $6.62 \mathrm{~b}$ \\
$\mathrm{M}_{2}$ & $32.33 \mathrm{a}$ & $60.67 \mathrm{a}$ & $76.74 \mathrm{a}$ & $82.15 \mathrm{a}$ & $6.84 \mathrm{a}$ & 6.67 & $6.84 \mathrm{a}$ & $7.07 \mathrm{a}$ \\
$\mathrm{M}_{3}$ & $31.17 \mathrm{~b}$ & $57.84 \mathrm{~b}$ & $74.46 \mathrm{~b}$ & $80.91 \mathrm{~b}$ & $6.58 \mathrm{ab}$ & 6.58 & $6.58 \mathrm{~b}$ & $6.76 \mathrm{~b}$ \\
$\mathrm{M}_{4}$ & $27.27 \mathrm{~d}$ & $50.28 \mathrm{~d}$ & $71.24 \mathrm{~d}$ & $76.79 \mathrm{~d}$ & $6.03 \mathrm{c}$ & 5.83 & $6.03 \mathrm{~d}$ & $5.83 \mathrm{c}$ \\
$\mathrm{CV}(\%)$ & 6.05 & 8.94 & 2.17 & 2.75 & 7.14 & 12.74 & 7.25 & 11.42 \\
Level of & & & & & & & & $*$ \\
significance & $* *$ & $* *$ & $* *$ & $* *$ & $* *$ & NS & $* *$ & $* *$ \\
\hline
\end{tabular}

Here, in a column, figures with same letters or without letter do not differ significantly whereas figures with dissimilar letter differ significantly as per DMRT. $\mathrm{M}_{1}=$ Traditional flooding, $\mathrm{M}_{2}=$ Non-flooded rice straw mulching, $\mathrm{M}_{3}=$ Non-flooded water hyacinth mulching, $\mathrm{M}_{4}=$ Non-flooded no mulching, ${ }^{* *}=$ Significant at $1 \%$ level of probability, NS=Non significant, DAT= Days after transplanting

Table 3. Interaction effect of variety and water management system on growth of boro rice at different dates after transplanting (DAT)

\begin{tabular}{ccccccccc}
\hline $\begin{array}{c}\text { Interaction } \\
\text { combination }\end{array}$ & \multicolumn{5}{c}{ Plant height $(\mathbf{c m})$} & \multicolumn{5}{c}{ No of tillers hill $^{-1}$} \\
\cline { 2 - 9 } & $\mathbf{2 5}$ & $\mathbf{5 0}$ & $\mathbf{7 5}$ & $\mathbf{1 0 0}$ & $\mathbf{2 5}$ & $\mathbf{5 0}$ & $\mathbf{7 5}$ & $\mathbf{1 0 0}$ \\
& $\mathbf{D A T}$ & $\mathbf{D A T}$ & $\mathbf{D A T}$ & $\mathbf{D A T}$ & $\mathbf{D A T}$ & $\mathbf{D A T}$ & DAT & DAT \\
\hline $\mathrm{V}_{1} \mathrm{M}_{1}$ & 28.60 & 52.67 & 71.04 & $77.94 \mathrm{f}$ & 6.49 & 6.45 & 6.49 & 6.40 \\
$\mathrm{~V}_{1} \mathrm{M}_{2}$ & 30.64 & 59.45 & 76.10 & $82.45 \mathrm{~d}$ & 6.78 & 6.67 & 6.78 & 6.80 \\
$\mathrm{~V}_{1} \mathrm{M}_{3}$ & 29.62 & 55.45 & 72.74 & $80.63 \mathrm{de}$ & 6.71 & 6.71 & 6.71 & 6.47 \\
$\mathrm{~V}_{1} \mathrm{M}_{4}$ & 26.19 & 50.12 & 70.53 & $79.26 \mathrm{ef}$ & 6.26 & 5.93 & 6.26 & 5.93 \\
$\mathrm{~V}_{2} \mathrm{M}_{1}$ & 37.37 & 60.67 & 78.63 & $89.29 \mathrm{~b}$ & 6.70 & 6.67 & 6.70 & 7.33 \\
$\mathrm{~V}_{2} \mathrm{M}_{2}$ & 40.81 & 68.00 & 81.56 & $94.00 \mathrm{a}$ & 7.45 & 7.33 & 7.45 & 7.87 \\
$\mathrm{~V}_{2} \mathrm{M}_{3}$ & 38.59 & 66.60 & 80.11 & $92.67 \mathrm{a}$ & 7.08 & 7.08 & 7.08 & 7.50 \\
$\mathrm{~V}_{2} \mathrm{M}_{4}$ & 35.69 & 55.18 & 76.74 & $85.12 \mathrm{c}$ & 6.58 & 6.27 & 6.58 & 6.27 \\
$\mathrm{~V}_{3} \mathrm{M}_{1}$ & 23.28 & 48.78 & 68.08 & $68.67 \mathrm{~g}$ & 5.85 & 5.97 & 5.85 & 6.13 \\
$\mathrm{~V}_{3} \mathrm{M}_{2}$ & 25.53 & 54.57 & 72.55 & $70.01 \mathrm{~g}$ & 6.30 & 6.00 & 6.30 & 6.53 \\
$\mathrm{~V}_{3} \mathrm{M}_{3}$ & 25.31 & 51.49 & 70.53 & $69.42 \mathrm{~g}$ & 5.97 & 5.97 & 5.97 & 6.30 \\
$\mathrm{~V}_{3} \mathrm{M}_{4}$ & 19.94 & 45.55 & 66.45 & $66.00 \mathrm{~h}$ & 5.25 & 5.29 & 5.25 & 5.29 \\
$\mathrm{CV}(\%)$ & 6.05 & 8.94 & 2.17 & 2.75 & 7.14 & 12.74 & 7.25 & 11.42 \\
Level of & & & & & & & & \\
significance & $\mathrm{NS}$ & $\mathrm{NS}$ & $\mathrm{NS}$ & $* *$ & $\mathrm{NS}$ & $\mathrm{NS}$ & $\mathrm{NS}$ & NS \\
\hline
\end{tabular}

Here, in a column, figures with same letters or without letter do not differ significantly whereas figures with dissimilar letter differ significantly as per DMRT. $\mathrm{V}_{1}=\mathrm{BRRI}$ dhan28, $\mathrm{V}_{2}=\mathrm{BRRI}$ dhan29, $\mathrm{V}_{3}=$ Binadhan-14, $\mathrm{M}_{1}=$ Traditional flooding, $\mathrm{M}_{2}=$ Non-flooded rice straw mulching, $\mathrm{M}_{3}=$ Non-flooded water hyacinth mulching, $\mathrm{M}_{4}=$ Nonflooded no mulching, $* *=$ Significant at $1 \%$ level of probability, $*=5 \%$ level of significance, NS $==$ Non significant, DAT=Days after transplanting 
Table 4. Effect of variety on shoot and root dry weight of boro rice at different days after transplanting (DAT)

\begin{tabular}{ccccc|cccc}
\hline \multirow{2}{*}{ Variety } & \multicolumn{4}{c}{ Shoot weight $(\mathbf{g})$} & \multicolumn{4}{c}{ Root weight $(\mathbf{g})$} \\
\cline { 2 - 9 } & $\mathbf{2 5}$ & $\mathbf{5 0}$ & $\mathbf{7 5}$ & $\mathbf{1 0 0}$ & $\mathbf{2 5}$ & $\mathbf{5 0}$ & $\mathbf{7 5}$ & $\mathbf{1 0 0}$ \\
& DAT & DAT & DAT & DAT & DAT & DAT & DAT & DAT \\
\hline V1 & $0.59 \mathrm{~b}$ & $5.50 \mathrm{~b}$ & $18.54 \mathrm{~b}$ & $22.58 \mathrm{~b}$ & $0.45 \mathrm{~b}$ & $2.80 \mathrm{~b}$ & $6.73 \mathrm{~b}$ & $9.22 \mathrm{ab}$ \\
V2 & $0.70 \mathrm{a}$ & $6.44 \mathrm{a}$ & $21.52 \mathrm{a}$ & $26.38 \mathrm{a}$ & $0.57 \mathrm{a}$ & $3.57 \mathrm{a}$ & $8.28 \mathrm{a}$ & $11.00 \mathrm{a}$ \\
V3 & $0.49 \mathrm{c}$ & $4.64 \mathrm{c}$ & $13.42 \mathrm{c}$ & $16.06 \mathrm{c}$ & $0.38 \mathrm{c}$ & $2.11 \mathrm{c}$ & $5.55 \mathrm{c}$ & $7.58 \mathrm{~b}$ \\
CV (\%) & 3.63 & 2.80 & 1.85 & 1.95 & 3.46 & 3.67 & 2.98 & 18.60 \\
Level of & & & & & & & & $*$ \\
significance & $* *$ & $* *$ & $* *$ & $* *$ & $* *$ & $* *$ & $*$ & $*$ \\
\hline
\end{tabular}

Here, in a column, figures with same letters or without letter do not differ significantly whereas figures with dissimilar letter differ significantly as per DMRT, $\mathrm{V}_{1}=\mathrm{BRRI}$ dhan $28, \mathrm{~V}_{2}=\mathrm{BRRI}$ dhan $29, \mathrm{~V}_{3}=$ Binadhan-14, ${ }^{* *}=$ Significant at $1 \%$ level of probability, ${ }^{*}=$ Significant at $5 \%$ level of probability, DAT=Days after transplanting

Table 5. Effect of water management system on shoot and root dry weight of boro rice at different days after transplanting (DAT)

\begin{tabular}{ccccc|cccc}
\hline & \multicolumn{4}{c|}{ Shoot weight (g) } & \multicolumn{4}{c}{ Root weight (g) } \\
\cline { 2 - 9 } Variety & $\mathbf{2 5}$ & $\mathbf{5 0}$ & $\mathbf{7 5}$ & $\mathbf{1 0 0}$ & $\mathbf{2 5}$ & $\mathbf{5 0}$ & $\mathbf{7 5}$ & $\mathbf{1 0 0}$ \\
& DAT & DAT & DAT & DAT & DAT & DAT & DAT & DAT \\
\hline $\mathrm{M}_{1}$ & $0.55 \mathrm{c}$ & $5.11 \mathrm{c}$ & $16.38 \mathrm{c}$ & $19.68 \mathrm{c}$ & $0.42 \mathrm{c}$ & $2.49 \mathrm{c}$ & $6.23 \mathrm{c}$ & $8.45 \mathrm{bc}$ \\
$\mathrm{M}_{2}$ & $0.70 \mathrm{a}$ & $6.50 \mathrm{a}$ & $22.44 \mathrm{a}$ & $27.71 \mathrm{a}$ & $0.60 \mathrm{a}$ & $3.80 \mathrm{a}$ & $8.50 \mathrm{a}$ & $11.47 \mathrm{a}$ \\
$\mathrm{M}_{3}$ & $0.63 \mathrm{~b}$ & $5.89 \mathrm{~b}$ & $19.18 \mathrm{~b}$ & $23.49 \mathrm{~b}$ & $0.50 \mathrm{~b}$ & $3.02 \mathrm{~b}$ & $7.13 \mathrm{~b}$ & $9.97 \mathrm{ab}$ \\
$\mathrm{M}_{4}$ & $0.49 \mathrm{~d}$ & $4.59 \mathrm{~d}$ & $13.30 \mathrm{~d}$ & $15.81 \mathrm{~d}$ & $0.34 \mathrm{~d}$ & $2.00 \mathrm{~d}$ & $5.56 \mathrm{~d}$ & $7.18 \mathrm{c}$ \\
$\mathrm{CV}(\%)$ & 3.63 & 2.80 & 1.85 & 1.95 & 3.46 & 3.67 & 2.98 & 18.60 \\
Level of & & & & $* *$ & & & & $*$ \\
significance & $* *$ & $* *$ & $* *$ & $* *$ & $* *$ & $* *$ & $* *$ & $* *$ \\
\hline
\end{tabular}

Here, in a column, figures with same letters or without letter do not differ significantly whereas figures with dissimilar letter differ significantly as per DMRT, $\mathrm{M}_{1}=$ Traditional flooding, $\mathrm{M}_{2}=$ Non-flooded rice straw mulching, $\mathrm{M}_{3}=$ Non-flooded water hyacinth mulching, $\mathrm{M}_{4}=$ Non-flooded no mulching, ${ }^{* *}=$ Significant at $1 \%$ level of probability, DAT=Days after transplanting

At this date, highest plant height $(94.00 \mathrm{~cm})$ was obtained from the combination of BRRI dhan29 and non-flooded rice straw mulching $\left(\mathrm{V}_{2} \mathrm{M}_{2}\right)$ Dry matter of shoot hill $^{-1}$ and dry matter of root hill ${ }^{-1}$ were significantly influenced by interaction of variety and water management system at all sampling dates except 25 DAT for shoot dry weight and 100 DAT for root dry weight (Table 6). The highest dry matter of shoot hill ${ }^{-1}$
$(32.30 \mathrm{~g})$ and dry matter of root $\operatorname{hill}^{-1}(14.11 \mathrm{~g})$ at 100 DAT was obtained from the combination of BRRI dhan29 and non-flooded rice straw mulching $\left(\mathrm{V}_{2} \mathrm{M}_{2}\right)$ and the lowest dry matter of shoot hill ${ }^{-1}$ and dry matter of root hill ${ }^{-1}$ was obtained from the combination of Binadhan-14 and non-flooded no mulching $\left(\mathrm{V}_{3} \mathrm{M}_{4}\right)$ treatment at all sampling dates. 


\section{Yield and yield contributing characters of boro rice}

Effect of variety: Plant height was significantly influenced by variety at harvest. The highest plant height was observed from BRRI dhan29 and that of lowest was recorded from Binadhan-14 (Figure 1). The number of total tillers hill ${ }^{-1}$, number of effective tillers hill $^{-1}$, panicle length, number of grains panicle ${ }^{-1}, 1000$ grain weight, biological yield and harvest index of boro rice at maturity was significantly influenced by variety (Table 7). BRRI dhan29 produced the highest number of total tillers hill $^{-1}$ (7.24), number of effective tillers hill $^{-1}$ (5.62), panicle length (20.16), number of grains panicle $^{-1}$ (95.03), 1000 grain weight (20.74 g), biological yield (11.77 $\left.\mathrm{t} \mathrm{ha}^{-1}\right)$ and harvest index (43.79) among the varieties. Binadhan-14produced the lowest plant height number of total tillers hill ${ }^{-1}$, number of effective tillers hill $^{-1}$, panicle length, number of grains panicle $^{-1}$, 1000 grain weight, biological yield and harvest index among the varieties. These differences are mostly due to the genetic variation between these three rice varieties. Variable effect of variety on number of total tillers hill ${ }^{-1}$ was also reported by BINA (1998). Nuruzzaman et al. (2000) noticed that number of total tillers hill $^{-1}$ differed among the varieties. This finding corroborates with those reported by BINA (1998), Om et al. (1998) who stated that effective tillers hill ${ }^{-1}$ was varied with variety.

Table 6. Interaction effect of variety and water management system on shoot and root dry weight of boro rice at different days after transplanting (DAT)

\begin{tabular}{ccccc|cccc}
\hline $\begin{array}{c}\text { Treatment } \\
\text { combination }\end{array}$ & \multicolumn{4}{c|}{ Shoot weight (g) } & \multicolumn{4}{c}{ Root weight (g) } \\
\cline { 2 - 9 } & $\mathbf{2 5}$ & $\mathbf{5 0}$ & $\mathbf{7 5}$ & $\mathbf{1 0 0}$ & $\mathbf{2 5}$ & $\mathbf{5 0}$ & $\mathbf{7 5}$ & $\mathbf{1 0 0}$ \\
& $\mathbf{D A T}$ & $\mathbf{D A T}$ & $\mathbf{D A T}$ & $\mathbf{D A T}$ & $\mathbf{D A T}$ & $\mathbf{D A T}$ & $\mathbf{D A T}$ & DAT \\
\hline $\mathrm{V}_{1} \mathrm{M}_{1}$ & 0.54 & $5.03 \mathrm{f}$ & $17.51 \mathrm{~g}$ & $20.76 \mathrm{f}$ & $0.40 \mathrm{~g}$ & $2.40 \mathrm{f}$ & $6.13 \mathrm{f}$ & 8.40 \\
$\mathrm{~V}_{1} \mathrm{M}_{2}$ & 0.70 & $6.53 \mathrm{c}$ & $22.11 \mathrm{c}$ & $28.70 \mathrm{~b}$ & $0.60 \mathrm{c}$ & $3.83 \mathrm{~b}$ & $8.20 \mathrm{c}$ & 11.30 \\
$\mathrm{~V}_{1} \mathrm{M}_{3}$ & 0.62 & $5.90 \mathrm{~d}$ & $19.63 \mathrm{e}$ & $24.23 \mathrm{~d}$ & $0.48 \mathrm{e}$ & $2.97 \mathrm{~cd}$ & $7.20 \mathrm{~d}$ & 9.88 \\
$\mathrm{~V}_{1} \mathrm{M}_{4}$ & 0.48 & $4.52 \mathrm{~g}$ & $14.90 \mathrm{i}$ & $16.63 \mathrm{~h}$ & $0.31 \mathrm{i}$ & $2.00 \mathrm{~h}$ & $5.40 \mathrm{gh}$ & 7.30 \\
$\mathrm{~V}_{2} \mathrm{M}_{1}$ & 0.67 & $6.00 \mathrm{~d}$ & $20.00 \mathrm{~d}$ & $24.08 \mathrm{~d}$ & $0.52 \mathrm{~d}$ & $3.07 \mathrm{c}$ & $7.33 \mathrm{~d}$ & 9.77 \\
$\mathrm{~V}_{2} \mathrm{M}_{2}$ & 0.81 & $7.55 \mathrm{a}$ & $27.10 \mathrm{a}$ & $32.30 \mathrm{a}$ & $0.71 \mathrm{a}$ & $4.73 \mathrm{a}$ & $10.63 \mathrm{a}$ & 14.11 \\
$\mathrm{~V}_{2} \mathrm{M}_{3}$ & 0.74 & $6.83 \mathrm{~b}$ & $22.86 \mathrm{~b}$ & $28.17 \mathrm{c}$ & $0.62 \mathrm{~b}$ & $3.90 \mathrm{~b}$ & $8.60 \mathrm{~b}$ & 12.03 \\
$\mathrm{~V}_{2} \mathrm{M}_{4}$ & 0.59 & $5.37 \mathrm{e}$ & $16.10 \mathrm{~h}$ & $20.97 \mathrm{f}$ & $0.43 \mathrm{f}$ & $2.60 \mathrm{e}$ & $6.57 \mathrm{e}$ & 8.10 \\
$\mathrm{~V}_{3} \mathrm{M}_{1}$ & 0.45 & $4.30 \mathrm{~h}$ & $11.62 \mathrm{j}$ & $14.20 \mathrm{i}$ & $0.35 \mathrm{~h}$ & $2.00 \mathrm{~h}$ & $5.23 \mathrm{~h}$ & 7.19 \\
$\mathrm{~V}_{3} \mathrm{M}_{2}$ & 0.59 & $5.41 \mathrm{e}$ & $18.10 \mathrm{f}$ & $22.13 \mathrm{e}$ & $0.50 \mathrm{de}$ & $2.83 \mathrm{~d}$ & $6.67 \mathrm{e}$ & 9.00 \\
$\mathrm{~V}_{3} \mathrm{M}_{3}$ & 0.52 & $4.93 \mathrm{f}$ & $15.04 \mathrm{i}$ & $18.07 \mathrm{~g}$ & $0.40 \mathrm{~g}$ & $2.20 \mathrm{~g}$ & $5.60 \mathrm{~g}$ & 8.00 \\
$\mathrm{~V}_{3} \mathrm{M}_{4}$ & 0.38 & $3.90 \mathrm{i}$ & $8.90 \mathrm{k}$ & $9.83 \mathrm{j}$ & $0.27 \mathrm{j}$ & $1.40 \mathrm{i}$ & $4.70 \mathrm{i}$ & 6.13 \\
$\mathrm{CV}(\%)$ & 3.63 & 2.80 & 1.85 & 1.95 & 3.46 & 3.67 & 2.98 & 18.60 \\
Level of & & & & & & & & \\
significance & $\mathrm{NS}$ & $* *$ & $* *$ & $* *$ & $* *$ & $* *$ & $* *$ & $\mathrm{NS}$
\end{tabular}

Here, in a column, figures with same letters or without letter do not differ significantly whereas figures with dissimilar letter differ significantly as per DMRT. $\mathrm{V}_{1}=\mathrm{BRRI}$ dhan28, $\mathrm{V}_{2}=\mathrm{BRRI}$ dhan29, $\mathrm{V}_{3}=\mathrm{Binadhan}-14, \mathrm{M}_{1}=$ Traditional flooding, $\mathrm{M}_{2}=$ Non-flooded rice straw mulching, $\mathrm{M}_{3}=$ Non-flooded water hyacinth mulching, $\mathrm{M}_{4}=$ Nonflooded no mulching, $* *=$ Significant at $1 \%$ level of probability, NS $=$ Non significant and DAT $=$ Days after transplanting 
Table 7. Effect of variety on yield and yield contributing characters of boro rice

\begin{tabular}{|c|c|c|c|c|c|c|c|c|}
\hline Variety & $\begin{array}{c}\text { Total } \\
\text { tillers } \\
\text { hill }^{-1}\end{array}$ & $\begin{array}{c}\text { No. of } \\
\text { effective } \\
\text { tillers } \\
\text { hill }^{-1}\end{array}$ & $\begin{array}{c}\text { No. of } \\
\text { non- } \\
\text { effective } \\
\text { tillers } \\
\text { hill }^{-1}\end{array}$ & $\begin{array}{c}\text { Panicle } \\
\text { length } \\
\mathbf{( c m})\end{array}$ & $\begin{array}{c}\text { No. of } \\
\text { grains } \\
\text { panicle }^{-1}\end{array}$ & $\begin{array}{c}\text { Wt. of } \\
\mathbf{1 0 0 0} \\
\text { grain }^{(\mathbf{g})}\end{array}$ & $\begin{array}{c}\text { Biological } \\
\text { yield } \\
\mathbf{( t ~ h a ~}^{-\mathbf{1}} \mathbf{)}\end{array}$ & $\begin{array}{c}\text { Harvest } \\
\text { index } \\
\mathbf{( \% )}\end{array}$ \\
\hline $\mathrm{V}_{1}$ & $6.47 \mathrm{~b}$ & $5.12 \mathrm{~b}$ & 1.35 & $19.26 \mathrm{~b}$ & $90.34 \mathrm{~b}$ & $20.17 \mathrm{~b}$ & $10.77 \mathrm{~b}$ & $42.04 \mathrm{~b}$ \\
\hline $\mathrm{V}_{2}$ & $7.24 \mathrm{a}$ & $5.62 \mathrm{a}$ & 1.63 & $20.16 \mathrm{a}$ & $95.03 \mathrm{a}$ & $20.74 \mathrm{a}$ & $11.77 \mathrm{a}$ & $43.79 \mathrm{a}$ \\
\hline $\mathrm{V}_{3}$ & $6.06 \mathrm{c}$ & $4.69 \mathrm{c}$ & 1.37 & $18.28 \mathrm{c}$ & $74.77 \mathrm{c}$ & $19.27 \mathrm{c}$ & $9.30 \mathrm{c}$ & $40.18 \mathrm{c}$ \\
\hline $\mathrm{CV}(\%)$ & 11.40 & 8.98 & 44.00 & 2.32 & 2.24 & 3.54 & 3.80 & 5.54 \\
\hline $\begin{array}{c}\text { Level of } \\
\text { significance }\end{array}$ & $*$ & $* *$ & $\mathrm{NS}$ & $* *$ & $* *$ & $* *$ & $* *$ & $* *$ \\
\hline
\end{tabular}

Here, in a column, figures with same letters or without letter do not differ significantly whereas figures with dissimilar letter differ significantly as per DMRT. $\mathrm{V}_{1}=\mathrm{BRRI}$ dhan28, $\mathrm{V}_{2}=\mathrm{BRRI}$ dhan29, $\mathrm{V}_{3}=$ Binadhan-14, ** = Significant at $1 \%$ level of probability, ${ }^{*}=$ Significant at $5 \%$ level of probability, NS $==$ Non significant

Table 8. Effect of water management system on yield and yield contributing characters of boro rice

\begin{tabular}{|c|c|c|c|c|c|c|c|c|}
\hline $\begin{array}{c}\text { Water } \\
\text { management } \\
\text { system }\end{array}$ & $\begin{array}{c}\text { Total } \\
\text { tillers } \\
\text { hill }^{-1}\end{array}$ & $\begin{array}{c}\text { No. of } \\
\text { effective } \\
\text { tillers } \\
\text { hill }^{-1}\end{array}$ & $\begin{array}{c}\text { No. of } \\
\text { non } \\
\text { effective } \\
\text { tillers } \\
\text { hill }^{-1}\end{array}$ & $\begin{array}{c}\text { Panicle } \\
\text { Length } \\
(\mathrm{cm})\end{array}$ & $\begin{array}{c}\text { No. of } \\
\text { grains } \\
\text { panicle }^{-1}\end{array}$ & $\begin{array}{c}\text { Wt. of } \\
1000 \\
\text { grain(g) }\end{array}$ & $\begin{array}{c}\text { Biological } \\
\text { yield } \\
\left(\mathrm{t} \mathrm{ha}^{-1}\right)\end{array}$ & $\begin{array}{c}\text { Harvest } \\
\text { index } \\
(\%)\end{array}$ \\
\hline $\mathrm{M}_{1}$ & $6.62 \mathrm{~b}$ & $5.09 \mathrm{c}$ & 1.53 & $18.80 \mathrm{c}$ & $86.89 c$ & $19.94 \mathrm{c}$ & $9.85 \mathrm{c}$ & $43.31 \mathrm{a}$ \\
\hline $\mathrm{M}_{2}$ & $7.13 a$ & $5.50 \mathrm{a}$ & 1.63 & $20.24 a$ & $89.27 a$ & $20.69 a$ & $12.49 \mathrm{a}$ & $41.83 \mathrm{c}$ \\
\hline $\mathrm{M}_{3}$ & $6.76 \mathrm{~b}$ & $5.24 b$ & 1.51 & $19.63 b$ & $88.30 \mathrm{~b}$ & $20.29 b$ & $11.70 \mathrm{~b}$ & $42.80 \mathrm{~b}$ \\
\hline $\mathrm{M}_{4}$ & 5.85 & $4.73 \mathrm{~d}$ & 1.12 & $18.27 \mathrm{~d}$ & $82.39 \mathrm{~d}$ & $19.32 \mathrm{~d}$ & $8.42 \mathrm{~d}$ & $40.07 d$ \\
\hline CV (\%) & 11.40 & 8.98 & 44.00 & 2.32 & 2.24 & 3.54 & 3.80 & 5.54 \\
\hline $\begin{array}{c}\text { Level of } \\
\text { significance }\end{array}$ & $*$ & $* *$ & NS & $* *$ & $* *$ & $* *$ & $* *$ & $*$ \\
\hline
\end{tabular}

Similar results were reported by Srivastava and Thipathi, 1998. Varietal differences regarding the number of grains panicle ${ }^{-1}$ might be due to their differences in genetic constitution. Accordingly, the highest grain yield $\left(5.16 \mathrm{tha}^{-1}\right)$ and straw yield $(6.60 \mathrm{t}$ $\mathrm{ha}^{-1}$ ) was obtained from BRRI dhan29 (Figure 2).

Effect of water management system: Water management system had significant influenced on plant height, number of total tillers hill ${ }^{-1}$, number of effective tillers hill ${ }^{-1}$, panicle length, number of grains panicle ${ }^{-1}$, 1000 grain weight, grain yield, straw yield, biological yield and harvest index of boro rice. Non-flooded rice straw mulching produced the highest plant height $((82.15 \mathrm{~cm})$ at maturity where as non-flooded no mulching produced the lowest plant height (Figure 1). Highest number of total tillers hill ${ }^{-1}$ (7.13), number of effective tillers hill ${ }^{-1}$ (5.50), panicle length (20.24), number of grains panicle ${ }^{-1}$ (89.27), biological yield (12.49) and harvest index (43.31\%) was obtained from non-flooded rice straw mulching (Table 8). Nonflooded no mulching produced the lowest plant height, number of total tillers hill ${ }^{-1}$, number of effective tillers 
hill $^{-1}$, panicle length, number of grains panicle ${ }^{-1}$, biological yield and harvest index among the water management systems. Similarly highest grain yield $\left(5.24 \mathrm{t} \mathrm{ha}^{-1}\right)$ and straw yield $\left(7.24 \mathrm{t} \mathrm{ha}^{-1}\right)$ was recorded from non-flooded rice straw mulching (Figure 2).
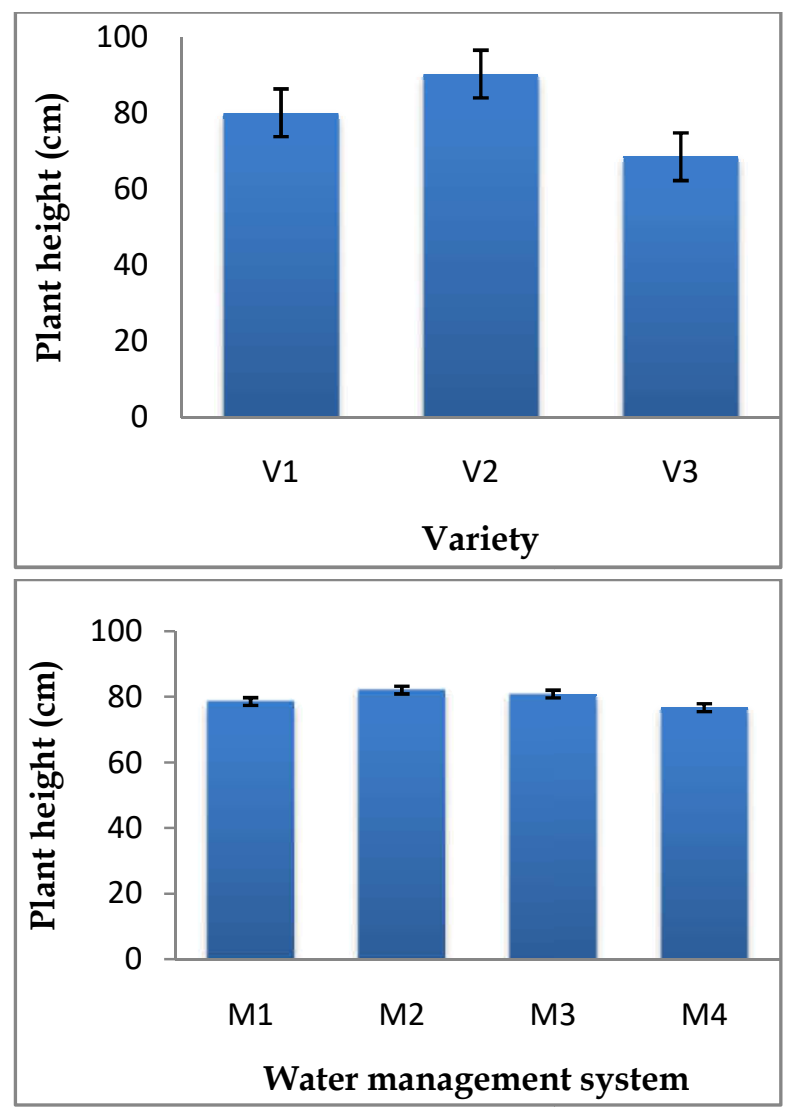

Figure 1. Effect of variety and water management system on plant height of boro rice

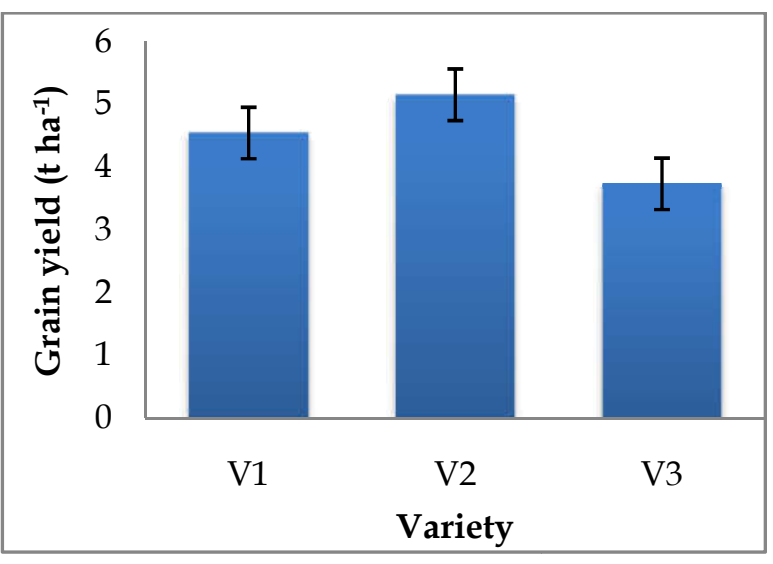

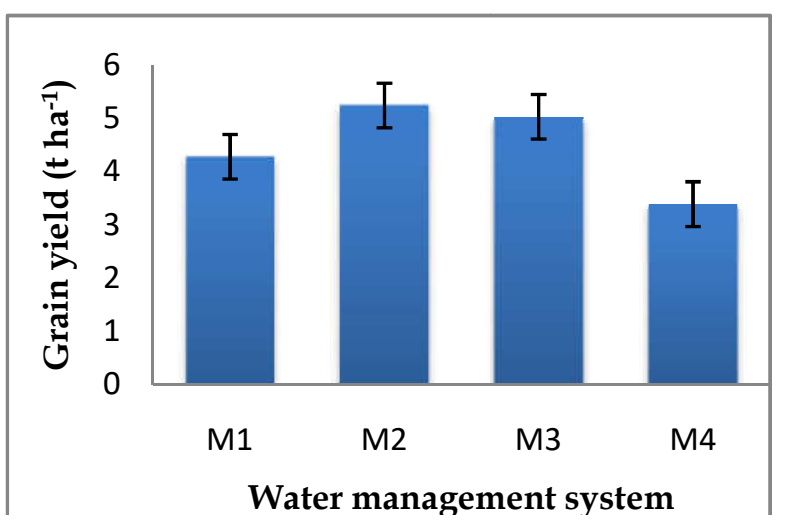

Figure 2. Effect of variety and water management system on grain yield of boro rice
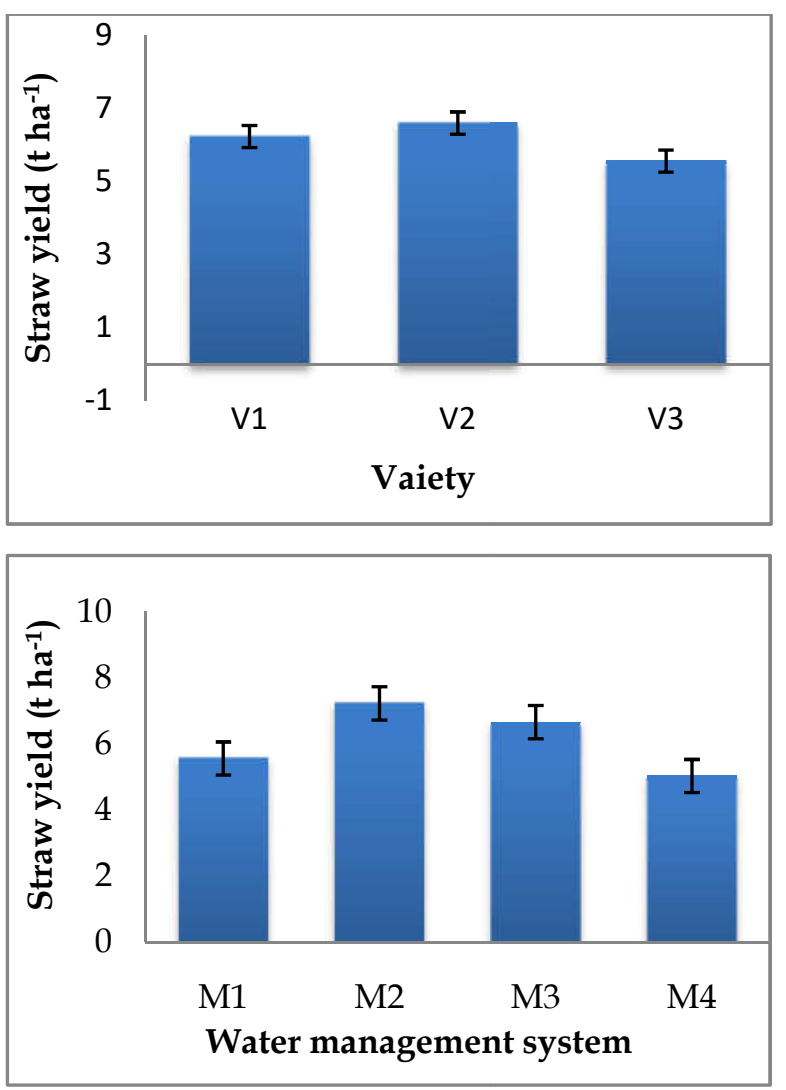

Figure 3. Effect of variety and water management system on straw yield of boro rice

Here, $\mathrm{V}_{1}=$ BRRI dhan28, $\mathrm{V}_{2}=$ BRRI dhan29, $\mathrm{V}_{3}=$ Binadhan-14, $\mathrm{M}_{1}=$ Traditional flooding, $\mathrm{M}_{2}=$ Nonflooded rice straw mulching, $\mathrm{M}_{3}=$ Non-flooded water hyacinth mulching and $\mathrm{M}_{4}=$ Non-flooded no mulching 
Table 9. Interaction effect of variety and water management system on yield and yield contributing characters of boro rice

\begin{tabular}{|c|c|c|c|c|c|c|c|c|c|c|c|}
\hline $\begin{array}{c}\text { Treatment } \\
\text { combination }\end{array}$ & $\begin{array}{c}\text { Plant } \\
\text { height } \\
(\mathrm{cm})\end{array}$ & $\begin{array}{l}\text { Total } \\
\text { tiller } \\
\text { hill }^{-1}\end{array}$ & $\begin{array}{c}\begin{array}{c}\text { No. of } \\
\text { effective }\end{array} \\
\text { tillers } \\
\text { hill }^{-1}\end{array}$ & $\begin{array}{c}\text { No. of } \\
\text { non- } \\
\text { effective } \\
\text { tillers } \\
\text { hill }^{-1}\end{array}$ & $\begin{array}{c}\text { Panicle } \\
\text { Length } \\
\text { (cm) }\end{array}$ & $\begin{array}{c}\text { No of } \\
\text { grains } \\
\text { panicle }^{-1}\end{array}$ & $\begin{array}{l}\text { Wt. of } \\
1000 \\
\text { grain } \\
\text { (g) }\end{array}$ & $\begin{array}{c}\text { Grain } \\
\text { yield } \\
\left(\text { tha }^{-1}\right)\end{array}$ & $\begin{array}{c}\text { Straw } \\
\text { yield } \\
\left(\text { tha }^{-1}\right)\end{array}$ & $\begin{array}{c}\text { Biologi } \\
\text { cal } \\
\text { yield } \\
\left(\mathrm{t} \mathrm{ha}^{-1}\right)\end{array}$ & $\begin{array}{c}\text { Harve } \\
\text { st } \\
\text { index } \\
(\%)\end{array}$ \\
\hline $\mathrm{V}_{1} \mathrm{M}_{1}$ & $77.94 \mathrm{f}$ & 6.40 & 5.00 & 1.40 & $18.80 \mathrm{c}$ & $91.66 \mathrm{~cd}$ & 20.03 & $4.34 \mathrm{~cd}$ & $5.63 d$ & $9.97 \mathrm{e}$ & 43.19 \\
\hline $\mathrm{V}_{1} \mathrm{M}_{2}$ & $82.45 \mathrm{~d}$ & 7.00 & 5.40 & 1.60 & $20.78 \mathrm{a}$ & $93.73 \mathrm{bcd}$ & 20.80 & $5.35 \mathrm{~b}$ & $7.31 \mathrm{a}$ & $12.66 \mathrm{~b}$ & 42.25 \\
\hline $\mathrm{V}_{1} \mathrm{M}_{3}$ & $80.63 \mathrm{de}$ & 6.47 & 5.20 & 1.27 & $19.40 \mathrm{~b}$ & $92.67 \mathrm{~cd}$ & 20.40 & $5.04 \mathrm{~b}$ & $6.82 \mathrm{~b}$ & $11.87 \mathrm{c}$ & 42.50 \\
\hline $\mathrm{V}_{1} \mathrm{M}_{4}$ & $79.26 \mathrm{ef}$ & 6.00 & 4.87 & 1.13 & $18.06 \mathrm{~d}$ & $83.28 \mathrm{e}$ & 19.45 & $3.46 \mathrm{f}$ & $5.13 \mathrm{e}$ & $8.59 \mathrm{f}$ & 40.21 \\
\hline $\mathrm{V}_{2} \mathrm{M}_{1}$ & $89.29 \mathrm{~b}$ & 7.33 & 5.73 & 1.60 & $19.60 \mathrm{~b}$ & $94.83 \mathrm{abc}$ & 20.70 & $4.86 b c$ & $6.25 \mathrm{c}$ & $11.11 \mathrm{~d}$ & 43.72 \\
\hline $\mathrm{V}_{2} \mathrm{M}_{2}$ & $94.00 \mathrm{a}$ & 7.87 & 6.00 & 1.87 & $21.12 \mathrm{a}$ & $98.08 \mathrm{a}$ & 21.27 & $6.06 \mathrm{a}$ & $7.73 a$ & $13.79 \mathrm{a}$ & 43.97 \\
\hline $\mathrm{V}_{2} \mathrm{M}_{3}$ & $92.67 \mathrm{a}$ & 7.50 & 5.73 & 1.77 & $20.73 \mathrm{a}$ & $96.67 \mathrm{ab}$ & 21.00 & $5.93 \mathrm{a}$ & $7.35 \mathrm{a}$ & $13.28 \mathrm{ab}$ & 44.64 \\
\hline $\mathrm{V}_{2} \mathrm{M}_{4}$ & $85.12 \mathrm{c}$ & 6.27 & 5.00 & 1.27 & $19.18 \mathrm{bc}$ & $90.53 \mathrm{~d}$ & 20.00 & $3.81 \mathrm{def}$ & $5.08 \mathrm{e}$ & $8.89 \mathrm{f}$ & 42.83 \\
\hline $\mathrm{V}_{3} \mathrm{M}_{1}$ & $68.67 \mathrm{~g}$ & 6.13 & 4.53 & 1.60 & $18.01 \mathrm{~d}$ & $74.17 \mathrm{f}$ & 19.10 & $3.64 \mathrm{ef}$ & $4.83 \mathrm{e}$ & $8.48 \mathrm{fg}$ & 43.03 \\
\hline $\mathrm{V}_{3} \mathrm{M}_{2}$ & $70.01 \mathrm{~g}$ & 6.53 & 5.10 & 1.43 & $18.81 \mathrm{c}$ & $76.00 \mathrm{f}$ & 20.00 & $4.31 \mathrm{~cd}$ & $6.69 \mathrm{~b}$ & $11.00 \mathrm{~d}$ & 39.25 \\
\hline $\mathrm{V}_{3} \mathrm{M}_{3}$ & $69.42 \mathrm{~g}$ & 6.30 & 4.80 & 1.50 & $18.75 \mathrm{c}$ & $75.55 \mathrm{f}$ & 19.48 & $4.10 \mathrm{de}$ & $5.83 \mathrm{~cd}$ & $9.94 \mathrm{e}$ & 41.25 \\
\hline $\mathrm{V}_{3} \mathrm{M}_{4}$ & $66.00 \mathrm{~h}$ & 5.29 & 4.33 & 0.95 & $17.57 \mathrm{e}$ & $73.37 \mathrm{f}$ & 18.52 & $2.90 \mathrm{~g}$ & $4.90 \mathrm{e}$ & $7.80 \mathrm{~g}$ & 37.18 \\
\hline CV (\%) & 3.36 & 11.40 & 8.98 & 44.00 & 2.32 & 2.24 & 3.54 & 7.36 & 4.18 & 3.80 & 5.54 \\
\hline $\begin{array}{c}\text { Level of } \\
\text { significance }\end{array}$ & $* *$ & NS & NS & NS & $* *$ & $*$ & NS & $*$ & $* *$ & $* *$ & NS \\
\hline
\end{tabular}

Here, in a column, figures with same letters or without letter do not differ significantly whereas figures with dissimilar letter differ significantly as per DMRT. $\mathrm{V}_{1}$ = BRRI dhan28, $\mathrm{V}_{2}=\mathrm{BRRI}$ dhan29, $\mathrm{V}_{3}=$ Binadhan-14, $\mathrm{M}_{1}$ $=$ Traditional flooding, $\mathrm{M}_{2}=$ Non-flooded rice straw mulching, $\mathrm{M}_{3}=$ Non-flooded water hyacinth mulching, $\mathrm{M}_{4}=$ Non-flooded no mulching, $* *=$ Significant at $1 \%$ level of probability, $*=5 \%$ level of significance and NS $=$ Non significant

\section{Interaction effect of variety and water management} system: The effect of interaction between variety and water management system was significant for plant height, panicle length, number of grains panicle ${ }^{-1}$, grain yield, straw yield and biological yield (Table 9). The highest plant height $(94.00 \mathrm{~cm})$, panicle length $(21.12$ $\mathrm{cm})$, number of grains panicle ${ }^{-1}$ (98.08) grain yield $\left(6.06 \mathrm{t} \mathrm{ha}^{-1}\right)$, straw yield (7.73 $\mathrm{t}$ ha-), biological yield $\left(13.79 \mathrm{tha}^{-1}\right)$ and harvest index (43.97\%) was obtained from $\mathrm{V}_{2} \mathrm{M}_{2}$ (BRRI dhan $29 \times$ non-flooded rice straw mulching) treatment and the lowest plant height, panicle length, number of grains panicle ${ }^{-1}$, grain yield, straw yield, biological yield and harvest index was found in $\mathrm{V}_{3} \mathrm{M}_{4}$ (Binadhan-14 $\times$ non-flooded no mulching) treatment (Table 9).

\section{Conclusion}

It was found that variety and water management system had significant effect on grain yield. BRRI dhan29 performed the best in respect of grain yield at non-flooded rice straw mulching treatment. BRRI dhan29, BRRI dhan28, and Binadhan-14produced the grain yields of $6.06,5.35$ and $4.31 \mathrm{tha}^{-1}$ respectively at non-flooded rice straw mulching treatment. It can be concluded that BRRI dhan29 with non-flooded rice straw mulching treatment was found to be the best possible combination for achieving higher grain yield.

\section{References}

BBS (Bangladesh Bureau of Statistics) 2011. Statistical Year book of Bangladesh. Bangladesh. Stat. Div., Minis. Plan., Govt. People's Repubic of 
Bangladesh, Dhaka. p.136-140.

BINA (Bangladesh Institute of Nuclear Agriculture). 1988. Annual Report for 1985-86. Bangladesh Inst. Nucl.Agr., P.O. Box No. 4, Mymensingh. p. 140-141.

BRRI. (Bangladesh Rice Research Institute) 2011.Annual Report for 1999. Bangladesh Rice Res. Inst., Joydebpur, Gazipur. p. 3-38.

De Silva CS, Weatherhead EK, Knox JW, RodriguezDiaz JA (2007). Predicting the impacts of climate change-A case study of paddy irrigation water requirement in Sri Lanka. Agril. Water. Mgt,93:19-29.

Gomez KA, Gomez AA (1984). Statistical Procedure nd

for Agricultural Research.2 Edn. A Wiley Interscience Publication, John Wiley and Sons, New York. p. 207-215.

Krisna K (2002). An Evaluating of Madagascar System of Rice production in amanSeason with Three High Potential Rice Varieties. MS Thesis, Dept. Agron., Bangladesh Agril. Univ., Mymensingh. p.98.

Nuruzzaman M, Yamamoto Y, Nitta Y, Yoshida Y, Miyazaki A (2000). Varietal differences in tillering ability of fourteen Japonica and Indica rice varieties. Soil Sci. Plant Nutri. 46(2): 381391.

Om H, Dhiman SD, Nandal DP, Verma SL (1998). Effect of method of nursery raising and nitrogen on growth and yield of hybrid rice (Oryza sativa).Indian J. Agron. 43(1): 68-70.

Sekhon GS, Abrol IP, Bhumbla DK (1986) Wheat growth and yield as affected by irrigation in a Hissar sandy loam soil sandy loam soil in: Proc. Symp. Water Mgt. Udaipur: 127-138.

Strivastava OK, Tripathi RS (1998). Response of hybrid and composite rice to number of seedling and planting genometry. Ann. Agril. Res. Newsl. 235-236.

Tisdale SL, Nelson W, Beaton, JD (1985). Soil fertilizer and fertilizers Macmillan Publishers, New York. p. 107-510.

UNDP, FAO (1998). Land Resources Appraisal of Bangladesh for Agricultural Development. Report 2. Agro-ecological Regions of Bangladesh.United Nation Develt.Prog. Food and Agric. Org. p. 212-221. 